\title{
Yposkesi : une plateforme industrielle pour le développement et la production de thérapies génique et cellulaire dans les maladies rares
}

\author{
Tuy Nga Brignol
}

En novembre 2016, l'AFM-Téléthon et le fonds d'investissement SPI « Société de Projets Industriels " géré par Bpifrance ont créé Yposkesi, du grec "Yposchesi " qui signifie la promesse, l'engagement. Localisé à Évry dans l'Essonne, c'est le premier acteur industriel pharmaceutique français dédié au développement et à la production de médicaments de thérapies génique et cellulaire pour les maladies rares.

La seule plateforme industrielle française dédiée aux médicaments de thérapies innovantes

Pour l'AFM-Téléthon, la création d'Yposkesi est l'étape indispensable pour atteindre son objectif qui n'a jamais changé : GUÉRIR. Constituée de plus de 120 experts de la bioproduction, cette plateforme industrielle est déjà dotée d'un premier bâtiment de production de $5000 \mathrm{~m}^{2}$ (anciennement Généthon Bioprod $^{1}$ ). Elle vise le leadership européen, avec pour 2021, un effectif de près de 300 ingénieurs, pharmaciens, techniciens et experts sur trois bâtiments de production totalisant $13000 \mathrm{~m}^{2}$.

L'AFM-Téléthon apporte le savoir-faire développé par ses laboratoires Généthon et CECS/I-stem, ainsi que leurs capacités de productions déjà existantes, et investira 37,5 millions d'euros d'ici 2022. Le fonds SPI "Société de Projets Industriels " investira au total 84 millions d'euros dans le cadre du Programme d'Investissement d'Avenir.

La société Yposkesi est gouvernée par l'AFM-Téléthon de façon majoritaire et par Bpifrance via le fonds SPI géré pour le compte de l'État. En mars dernier, Yposkesi a annoncé la nomination d'Alain Lamproye comme Directeur général. Avec plus de 25 ans d'expérience en Europe et aux États-Unis dans le domaine de la bioproduction industrielle, Alain Lamproye fera bénéficier Yposkesi de son expertise unique dans le management de la bioproduction et du développement pharmaceutique de produits de thérapies génique et cellulaire.
Une capacité de production importante de lots thérapeutiques

Yposkesi répond aux besoins des laboratoires de l'AFM-Téléthon (Généthon et CECS/I-Stem) ainsi qu'à ceux des acteurs académiques et industriels des biotechnologies pour les essais cliniques ou pour une diffusion post-AMM. Les premiers lots thérapeutiques produits par Yposkesi sont notamment des lots de thérapie génique pour des essais cliniques concernant la dystrophie musculaire de Duchenne, des maladies du système immunitaire comme le syndrome de Wiskott-Aldrich et la granulomatose septique chronique, et des maladies du sang comme l'anémie de Fanconi. En thérapie cellulaire, les premières productions d'Yposkesi concerneront les rétinites pigmentaires et les ulcères drépanocytaires dès que les capacités de production seront disponibles en 2018.

À l'horizon 2021, il est prévu chaque année une production de dizaines de lots de médicaments de thérapie génique et cellulaire pour traiter quelques dizaines à quelques milliers de patients selon les pathologies.

Yposkesi a pour ambition la mise à la disposition des malades des premiers traitements et leur commercialisation à un prix juste et maîtrisé, conformément à l'engagement de l'AFM-Téléthon et à la stratégie d'intérêt général voulue par ses fondateurs.

Yposkesi: an industrial platform for the development and production of gene and cell therapies in rare diseases
Tuy Nga Brigno
AFM-Téléthon tnbrignol@afm-telethon.fr

\section{${ }^{1}$ Les Cahiers de 2011, p. 45. \\ NOTE}


Yposkesi : deux partenaires, une même stratégie d'intérêt général AFM-Téléthon

Depuis le premier Téléthon en 1987, l'AFM-Téléthon a consacré plus de 1,2 milliard d'euros à l'innovation thérapeutique au bénéfice des personnes atteintes de maladies rares. Aujourd'hui, elle a développé une capacité unique pour concevoir des biothérapies innovantes et amener les premières preuves d'efficacité chez les patients à travers en particulier ses laboratoires d'excellence regroupés au sein de l'Institut des Biothérapies de maladies rares, notamment Généthon pour la thérapie génique des maladies rares et CECS/I-stem pour la thérapie cellulaires à partir des cellules souches pluripotentes. www.afm-telethon.fr

\section{Société de projets industriels SPI de Bpifrance}

Le fonds SPI, géré par Bpifrance pour le compte de l'État dans le cadre du Programme d'Investissements d'Avenir, a vocation à permettre aux projets industriels les plus porteurs de perspectives d'activité et d'emplois pour les filières industrielles de trouver un appui à leur développement. Il constitue ainsi l'un des leviers financiers de la Nouvelle France Industrielle.

Filiale de la Caisse des Dépôts et de l'État, Bpifrance accompagne les entreprises, de l'amorçage jusqu'à la cotation en bourse, en crédit, en garantie et en fonds propre. Bpifrance assure en outre des services d'accompagnement et de soutien renforcé à l'innovation, à la croissance externe et à l'export, en partenariat avec Business France et Coface.

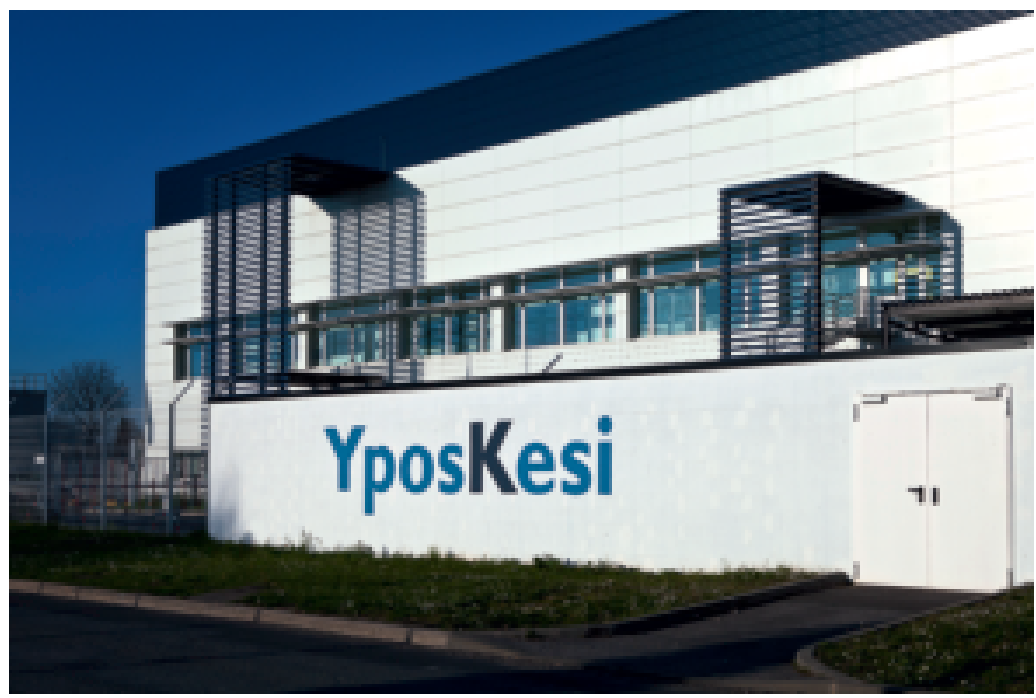

Bâtiment Yposkesi (๑) Christophe Hargoues) 